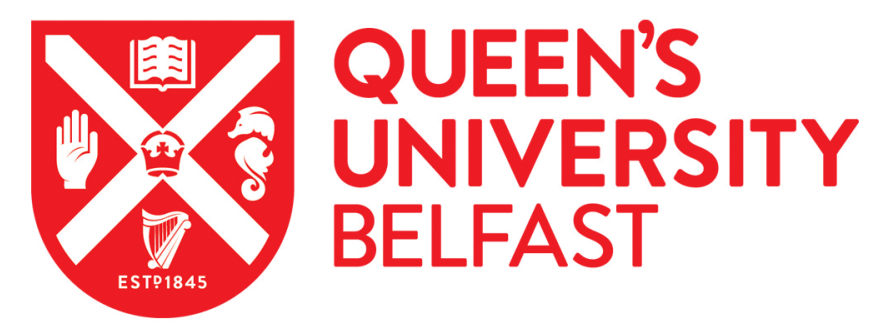

\title{
An integrated approach for real-time model-based state-of-charge estimation of lithium-ion batteries
}

Zhang, C., Li, K., Pei, L., \& Zhu, C. (2015). An integrated approach for real-time model-based state-of-charge estimation of lithium-ion batteries. Journal of Power Sources, 283, 24-36.

https://doi.org/10.1016/j.jpowsour.2015.02.099

Published in:

Journal of Power Sources

Document Version:

Peer reviewed version

Queen's University Belfast - Research Portal:

Link to publication record in Queen's University Belfast Research Portal

\footnotetext{
Publisher rights

(c) 2015 Elsevier. This manuscript version is made available under the CC-BY-NC-ND 4.0 license http://creativecommons.org/licenses/by-ncnd/4.0/ which

permits distribution and reproduction for non-commercial purposes, provided the author and source are cited.
}

\section{General rights}

Copyright for the publications made accessible via the Queen's University Belfast Research Portal is retained by the author(s) and / or other copyright owners and it is a condition of accessing these publications that users recognise and abide by the legal requirements associated with these rights.

Take down policy

The Research Portal is Queen's institutional repository that provides access to Queen's research output. Every effort has been made to ensure that content in the Research Portal does not infringe any person's rights, or applicable UK laws. If you discover content in the Research Portal that you believe breaches copyright or violates any law, please contact openaccess@qub.ac.uk. 


\section{Accepted Manuscript}

An Integrated Approach for Real-time Model-based State-of-Charge Estimation of Lithium-ion Batteries

Cheng Zhang, Kang Li, Lei Pei, Chunbo Zhu

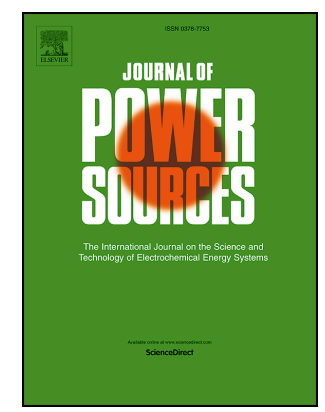

PII:

S0378-7753(15)00344-4

DOI:

10.1016/j.jpowsour.2015.02.099

Reference: POWER 20727

To appear in: Journal of Power Sources

Received Date: 22 November 2014

Revised Date: 4 February 2015

Accepted Date: 17 February 2015

Please cite this article as: C. Zhang, K. Li, L. Pei, C. Zhu, An Integrated Approach for Real-time Modelbased State-of-Charge Estimation of Lithium-ion Batteries, Journal of Power Sources (2015), doi: 10.1016/j.jpowsour.2015.02.099.

This is a PDF file of an unedited manuscript that has been accepted for publication. As a service to our customers we are providing this early version of the manuscript. The manuscript will undergo copyediting, typesetting, and review of the resulting proof before it is published in its final form. Please note that during the production process errors may be discovered which could affect the content, and all legal disclaimers that apply to the journal pertain. 


\title{
An Integrated Approach for Real-time Model-based State-of-Charge Estimation of Lithium-ion Batteries
}

\author{
Cheng Zhang ${ }^{\mathrm{a}}$, Kang $\mathrm{Li}^{\mathrm{a}}{ }^{\mathrm{*}}$, Lei $\mathrm{Pei}^{\mathrm{b}}$, Chunbo $\mathrm{Zhu}^{\mathrm{b}}$ \\ ${ }^{a}$ School of Electronics, Electrical Engineering and Computer Science, Queen's University \\ Belfast, 125 Stramillis Road, Ashby Building, Belfast, BT9 5AH, UK \\ ${ }^{b}$ School of Electrical Engineering and Automation, Harbin Institute of Technology, 92 \\ Xidazhi St., Harbin, 150001, China
}

\begin{abstract}
Lithium-ion batteries have been widely adopted in electric vehicles (EVs), and accurate state of charge (SOC) estimation is of paramount importance for the EV battery management system. Though a number of methods have been proposed, the SOC estimation for Lithium-ion batteries, such as LiFePo4 battery, however, faces two key challenges: the flat open circuit voltage (OCV) versus SOC relationship for some SOC ranges and the hysteresis effect. To address these problems, an integrated approach for real-time model-based SOC estimation of Lithium-ion batteries is proposed in this paper. Firstly, an autoregression model is adopted to reproduce the battery terminal behaviour, combined with a non-linear complementary model to capture the hysteresis effect. The model parameters, including linear parameters and non-linear parameters, are optimized off-line using a hybrid optimization method that combines a metaheuristic method (i.e., the teaching learning based optimization method) and the least square method. Secondly, using the trained model, two real-time model-based SOC estimation methods are presented, one based on the realtime battery OCV regression model achieved through weighted recursive least square method, and the other based on the state estimation using the extended Kalman filter method (EKF). To tackle the problem caused by the flat OCVversus-SOC segments when the OCV-based SOC estimation method is adopted,
\end{abstract}

*Corresponding author: k.li@qub.ac.uk (K. Li) 
a method combining the coulombic counting and the OCV-based method is proposed. Finally, modelling results and SOC estimation results are presented and analysed using the data collected from LiFePo4 battery cell. The results confirmed the effectiveness of the proposed approach, in particular the joint-EKF method.

Keywords: LiFePo4 battery, Real-time SOC estimation, Hysteresis effect, Extended Kalman Filter, Weighted Recursive Least square, Teaching Learning Based Optimization (TLBO) method

\section{Introduction}

Due to the imminent challenges of environment protection and the exhaustion of non-renewable fossil fuels, electric vehicles (EVs) and hybrid electric vehicles (HEVs) are rapidly gaining popularity worldwide in recent years as an effort of replacing the internal combustion engine (ICE) vehicles to improve the fuel efficiency and reduce the emissions in the transport sector. Many countries have proposed their national plans to increase the EV/HEV penetration in the coming decades [1]. The battery system is a key component in the EV/HEV system. Among different cell types, Lithium-ion batteries, such as LiFePo4 that is under investigation in this paper, are favoured power supplies for EVs and HEVs due to their high power and high energy densities, long service life, high efficiency and environmental-friendly figures [2. A battery management system (BMS) is essential in EV/HEV applications for safe and efficient operation where hundreds or even thousands of battery cells are connected in series/parallel configuration to fulfil the high power and high voltage needs of the vehicles [3]. One key functionality of the BMS is to estimate the state-ofcharge (SOC) of the battery, which is not directly measurable. SOC indicates the charge left in the battery available for further service, which determines the remaining range an EV can travel without re-charging the battery. Battery SOC indicator is similar to the fuel gauge in an ICE vehicle. Therefore accurate realtime SOC estimation is of great importance to prevent stranding halfway and to 
relieve the range anxiety. Further, SOC estimation can be used for preventing over-charging and over-discharging operations of the battery, thus reducing the harm caused to the battery. Some EVs require to cycle the battery within a 25 specific SOC range, e.g., $20 \%$ to $70 \%$, to achieve higher efficiency and longer service life, which again relies on accurate SOC estimation. On the other hand, inaccurate SOC estimation will result in an over-sized battery system, therefore a significant increase of the overall cost of EVs.

Another application of SOC estimation is for battery cell balancing. There are slight differences between different cells within the same pack, such as different cell capacity or internal impedance. As time goes by, this difference will become more and more significant [4]. The overall capacity of battery cells connected in series is limited by the cell with the least capacity, and without a balancing method this cell will be stressed more than other cells under the same working condition, leading to a deteriorating unbalancing problem. Therefore cell balancing is another essential functionality of the BMS, and the cell SOC can be used as an indicator for balancing the battery [3. There are other advantages brought by accurate SOC estimation, such as accurate available power estimation, and battery SOC estimation can also be used for developing power so and energy management strategies, etc.

Despite the demanding necessity, accurate real-time SOC estimation is not easy to acquire. First of all, all the estimation methods in the EV applications should be based on the on-board measured signals, such as the battery terminal voltage, load current and the temperature. Due to the high-voltage, high-current

45 and highly dynamic profile of the load, voltage and current measurements are often corrupted with noises. Besides, some SOC estimation methods, such as the open circuit voltage (OCV) based methods, are sensitive to the voltage measurement error. Secondly, the battery behaviour is highly non-linear and nonstationary, and some internal chemical reactions, such as the parasitic reaction, self-discharge and ageing process that affect the battery SOC, are extremely difficult to model.

Over the years, researchers have developed different SOC estimation meth- 
ods [5, 6, 7, 8, 9, 10, These methods can be generally divided into two groups: direct measurement methods and model-based estimation methods. Direct measurement methods, or model-free methods, estimate battery SOC by a directly measurable physical property, such as coulombic counting method (or Ah method) and OCV based methods. For model-based SOC estimation methods, a model is firstly built to reproduce the battery terminal behaviour. Then the battery SOC can be linked to one or several of the model parameters. After the model parameters are identified, the battery SOC can be inferred. Another approach is to model the battery behaviour using a state-space model with the battery SOC as one state, then different state estimation methods, such as Kalman Filter (KF) and Unscented Kalman Filter (UKF), can be used for SOC estimation. Direct measurement methods are generally open-loop methods. They 65 are easy to implement, but sensitive to current and voltage measurement errors. On the other hand, the model-based methods are generally close-loop methods and not sensitive to measurement errors, but they rely on an accurate battery model, which is difficult to acquire.

Further, the SOC estimation for Lithium-ion batteries faces two key challenges. Firstly, batteries like $\mathrm{LiFePo} 4$ show a flat OCV-versus-SOC curve within some SOC ranges, and therefore a small voltage measurement error can cause a large SOC estimation error for the OCV-based SOC estimation methods. Another difficulty is that the battery shows a hysteresis effect, i.e., the battery OCV depends on the direction of the load current, which needs to be considered during battery modelling and SOC estimation. To address these problems, an integrated approach for real-time model-based SOC estimation of Lithiumion batteries is proposed in this paper. The contributions of this paper are summarized as follows. Firstly, a new battery model is proposed, including an auto-regression relaxation model together with a non-linear complementary model to capture the hysteresis effect. Secondly, the model parameters are divided into two groups, namely the linear parameters and the non-linear parameters, and a hybrid optimization method that combines a meta-heuristic method (i.e., the teaching learning based optimization (TLBO) method) and the least 
square method is used to optimize the two distinctively different sets of parameters. This leads to a high modelling accuracy. Thirdly, based on the off-line trained model, two real-time SOC estimation methods are then proposed using the weighted recursive least square (WRLS) method and the Kalman Filter method, respectively. Finally, to tackle the problem caused by the flat OCVversus-SOC curve of Lithium-ion batteries, a new method combining coulombic counting method and OCV-based method is also proposed.

The rest of this paper is organized as follows. Section II presents a brief introduction to different SOC estimation methods, including direct measurement methods and model-based methods. The battery test system and the test data used in this paper are presented in section III. Then the auto-regression model is presented in section IV, together with the hysteresis model. The model parameters are optimized using TLBO and least square method. The modelling results are then presented. The two different model-based SOC estimation methods are given in section $\mathrm{V}$, and the SOC estimation results are analysed in section VI. Finally, section VII concludes this paper.

\section{Different SOC estimation methods}

\subsection{Direct measurement methods}

Based on the onboard measurable signals, i.e., battery terminal voltage and current, there are two popular direct measurement methods for SOC estimation, i.e., coulombic counting method (or Ah method, Ah stands for Ampere-hour, which is the unit of battery capacity) and OCV-based method.

\subsubsection{Ah method}

The Ah method is to integrate the discharging current to calculate the remaining charge in the battery, as follows.

$$
S O C(k)=S O C(0)-\frac{T}{C_{n}} \int_{0}^{k}\left(\eta * i(t)-S_{d}\right) d t
$$

where $S O C(0)$ is the initial SOC, $C_{n}$ the nominal capacity of the battery, $T$ is the sampling period, $i(t)$ is the load current at time $t, \eta$ is coulombic efficiency, 
and $S_{d}$ is the self-discharging rate. For LiFePo4 battery used in this experiment, $\eta>0.994$ under room temperature [11]; according to the manufacturer, the battery self-discharging rate is less than $5 \%$ per month. Therefore, $\eta=1$ and $S_{d}=0$ are assumed in this paper.

Based on the on-board measured current signals, it seems straightforward to apply Ah method for SOC estimation. However, this is an open-loop method, and is vulnerable to the current measurement error, especially the sensor drift error. Therefore, the Ah method needs to be calibrated periodically. Further, the initial SOC, $S O C(0)$, has to be determined accurately by other method. If the battery is fully charged periodically, then $S O C(0)$ can be calibrated to $100 \%$ after a fully charging procedure. However, during shadow cycle discharging when the battery is cycled within a limited SOC range, e.g., $30 \%-70 \%$, it is not easy to calibrate the initial SOC.

\subsection{2. $O C V$-based method}

The OCV method relies upon the relationship between battery OCV and SOC. Battery OCV is the battery terminal voltage when the battery internal equilibrium is reached in the absence of load. There exists a stable one-to-one relationship between battery OCV and SOC, if the temperature effect and hysteresis are not considered [12. Battery OCV voltage is slightly affected by the battery temperature [13. If the battery is operated under different temperature conditions, temperature effect on OCV should be taken into consideration [14. However, in this paper, the test is operated under constant temperature, and we assume that the battery pack temperature in EV/HEV is well controlled by the temperature management system, therefore temperature effect on OCV is not considered in this paper.

The hysteresis effect arises as the battery relaxes to a voltage value higher than the OCV for a given SOC after charging, and to a lower value than the OCV after discharging, even after sufficient relaxation time, as shown in Fig 1 . The battery OCV is taken as the mean of the charging OCV and discharging OCV, while the hysteresis is calculated as half the difference between the charging 
OCV and the discharging OCV [15]. The hysteresis effect in Li-ion batteries is generated due to the thermodynamic entropic effects, mechanical stress, and microscopic distortions within the active electrode materials during Lithium insertion/extraction [16]. LiFePo4 batteries show significant hysteresis effect [17, 18, as can be seen in Fig 1. Therefore, a hysteresis model is essential for accurate SOC estimation for $\mathrm{LiFePo} 4$ batteries when OCV based methods are used.

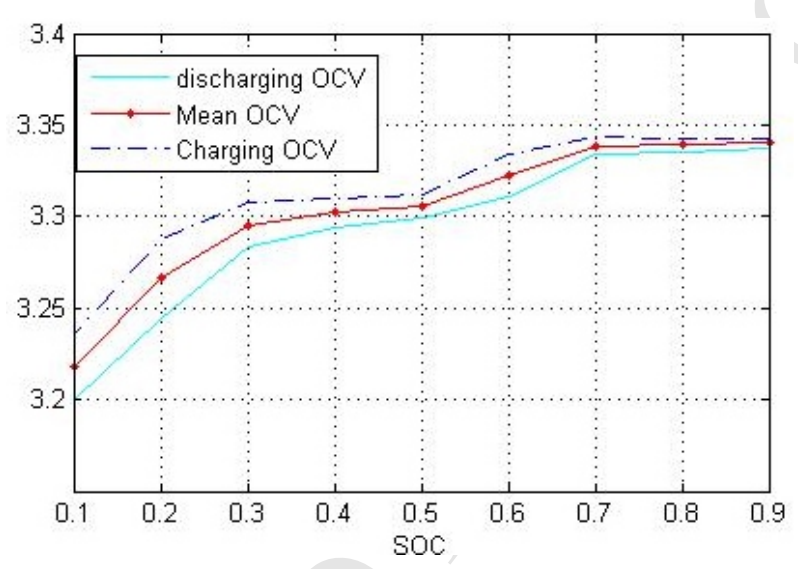

Figure 1: Hysteresis effect of the $\mathrm{LiFePo} 4$ cell used in this paper

As mentioned above, the Ah method needs to be calibrated periodically. Based on the onboard measured voltage signals, it is a natural choice to use battery OCV for calibration. However, it takes very long time (more than 1 hours) for the battery terminal voltage to stabilize. To tackle this problem, researchers have proposed several model-based methods to estimate battery OCV in real-time [19]. Another problem is that the LiFePo4 battery used in this experiment shows a very flat OCV-SOC curve during two SOC segments, one from $70 \%$ to $90 \%$ SOC and another from $30 \%$ to $50 \%$ SOC, as shown in Fig 1 making it extremely difficult to accurately estimate battery SOC using OCV-based method during these two SOC ranges. This effect will be detailed in the following section. 


\subsubsection{Other direct measurement methods}

There are other battery properties which can be used for SOC estimation, such as battery impedance [20, 21] and magnetism measurement [22]. Battery impedance is measured by generating a small AC current to flow through the battery under investigation, and the $\mathrm{AC}$ voltage response is recorded. Then the complex impedance of the battery can be calculated by a FFT analyser. The frequency of the AC current signal sweeps from $\mathrm{mHz}$ (sometimes $\mu \mathrm{Hz}$ ) to several $\mathrm{kHz}$ [23. The measured impedance in a range of frequency is referred to as the electrochemical impedance spectroscopy (EIS). However, specific equipments, such as a signal generator or potentiostat, are required by these methods, making it impractical for real-time EV applications.

\subsection{Model-based method}

There are different model-based SOC estimation methods that use different types of battery models. Electrochemical models (or physical model, white-box model) adopt physical laws, such as the porous electrode theory that governs the battery electrochemical processes, to describe battery behaviour. This is the most accurate battery model and can be used to estimate battery SOC [24, 25]. However, Electrochemical models are very complex and involve partial differential equations which are difficult to solve and analyse for real-time purposes. Besides, the model parameters are related to the electrochemical structure of the battery, which can be difficult to obtain. Based on the electrochemical model, researchers proposed reduced-order models by introducing more approximations, such as the volume averaging method, for model simplification and SOC estimation [24, 26, 27].

There are also different SOC estimation methods based on a simpler battery model, such as a linear equivalent circuit model (ECM) [28, 29]. A linear ECM uses a combination of electric elements, such as a voltage source, resistors and capacitors, to model the battery behaviour, as shown in Fig 2, where $O C V$ represents the battery OCV that depends on battery SOC, $R_{i}$ the internal resistance. The $R C$ networks are used to capture the battery relaxation effect. 
The ECM model enjoys a simple and interpretable structure, thus suitable for on-line applications.

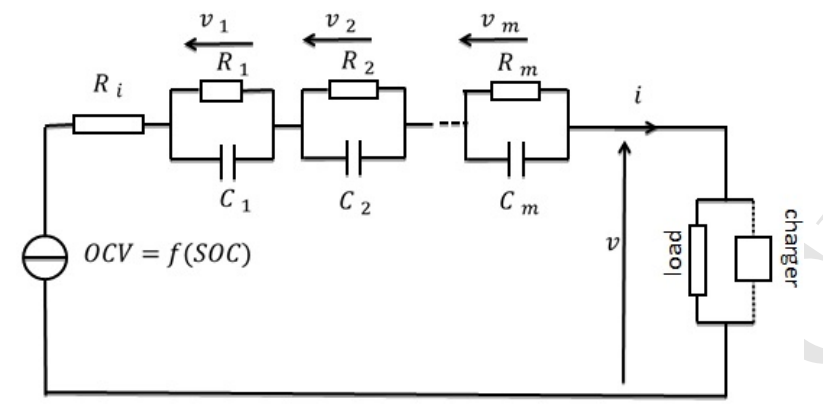

Figure 2: Linear equivalent circuit model

Many SOC estimation methods based on black-box models have been proposed, such as neural networks [30, 31, fuzzy logic [32, support vector machine [33, etc. There are also many combined methods for SOC estimation to make full use of the advantages of different methods 34 .

In this paper, we particularly investigate the SOC estimation of Lithium-ion batteries for real-time applications.

\section{Test Data}

A 5 - $\mathrm{Ah} \mathrm{LiFePo} 4$ battery was tested under room temperature $\left(25^{\circ} \mathrm{C}\right)$ with Arbin BT2000 battery test system. The voltage and current measurement accuracy are up to $0.02 \%$ full scale range (FSR) for low power applications and $0.05 \%$ FSR for high power applications. The temperature is maintained by a temperature chamber. Three different procedures were tested on the battery, namely, the Hybrid Pulse Power Characterization (HPPC) discharging test, HPPC charging test and the Federal Urban Drive Schedule (FUDS) test. The load current is positive for discharging and negative for charging.

During the HPPC discharging test, the battery is firstly fully charged to $100 \%$ SOC. Then the battery goes through ten discharging segments, as shown in Fig 3 One augmented part of the HPPC test data is shown in Fig 4 , where 
the starting two large current pulses, i.e., the first 10-second $25 \mathrm{~A}$ discharging current and the second 10-second 17.5 A charging current, are applied to test the battery's capacity of supporting large discharging and charging power, respectively. The following $5 \mathrm{~A}$ discharging pulse for 360 seconds reduces the battery SOC by $10 \%$.

The HPPC charging test data is shown in Fig 5 , with one segment augmented in Fig 6 As can be seen in Fig 6, the battery is firstly discharged using a 10second $25 \mathrm{~A}$ load current, and then charged by a 10-second $17.5 \mathrm{~A}$ current. The following 5 A charging current that lasts for 360 second is applied to increase the battery SOC by $10 \%$.

As can be seen in Fig 4 after the load current disappears, the battery terminal voltage relaxes gradually to a stable value, which is referred to as the relaxation effect. The battery is rested for one hour after each test segment, and the voltage at the end of the rest time is taken as the battery discharging OCV at that SOC point. The battery charging OCV is calculated in the same way using the HPPC charging data shown in Fig 6 . Battery OCV is taken as the mean value of the charging and discharging OCV, as shown in Fig 1 and in Table 1. As can be seen in Table 1, from $90 \%$ SOC to $70 \%$ SOC, the battery OCV is only reduced by $1.6 \mathrm{mV}$, while in practical $\mathrm{EV} / \mathrm{HEV}$ management system, the voltage measurement accuracy is usually about $5 \mathrm{mV}$. Therefore it is extremely difficult to achieve reliable SOC estimation using OCV-based methods during this SOC range. Therefore, the model-based SOC estimation is only considered during $10-70 \%$ SOC range.

The FUDS test shown in Fig 7 is to simulate the load profile of urban city driving, including acceleration and regenerative break, with one augmented segment shown in Fig 8

The test data under 10-90\% SOC range are shown in Fig 3 to Fig 7. 
Table 1: Battery mean OCV vs SOC

\begin{tabular}{llllll}
\hline $\mathrm{SOC} / \%$ & 10 & 20 & 30 & 40 & 50 \\
$\mathrm{OCV} / \mathrm{V}$ & 3.2176 & 3.2663 & 3.2955 & 3.3022 & 3.3056 \\
\hline $\mathrm{SOC} / \%$ & 60 & 70 & 80 & 90 & \\
$\mathrm{OCV} / \mathrm{V}$ & 3.3226 & 3.3385 & 3.3390 & 3.3401 & \\
\hline
\end{tabular}
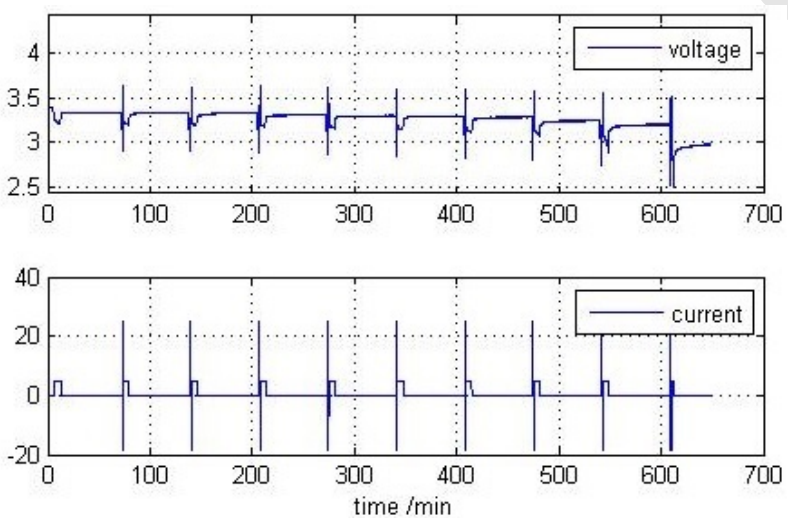

Figure 3: HPPC discharging test data
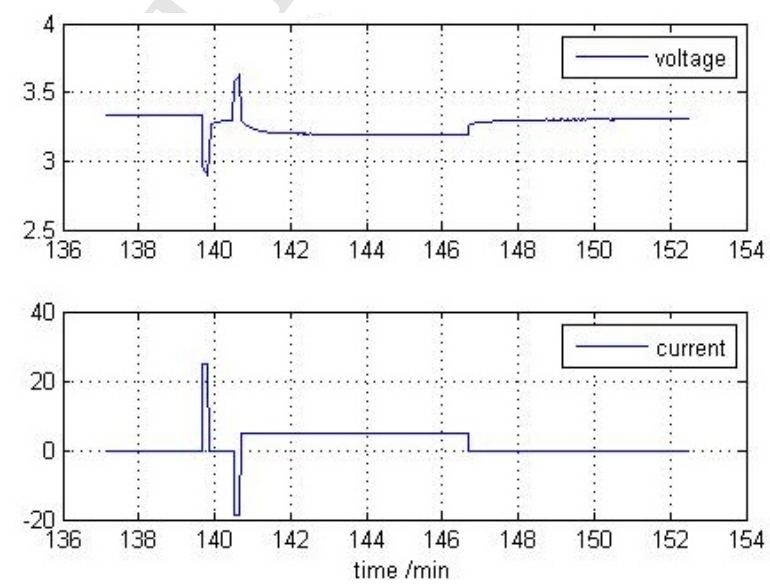

Figure 4: One segment of the HPPC discharging test data 

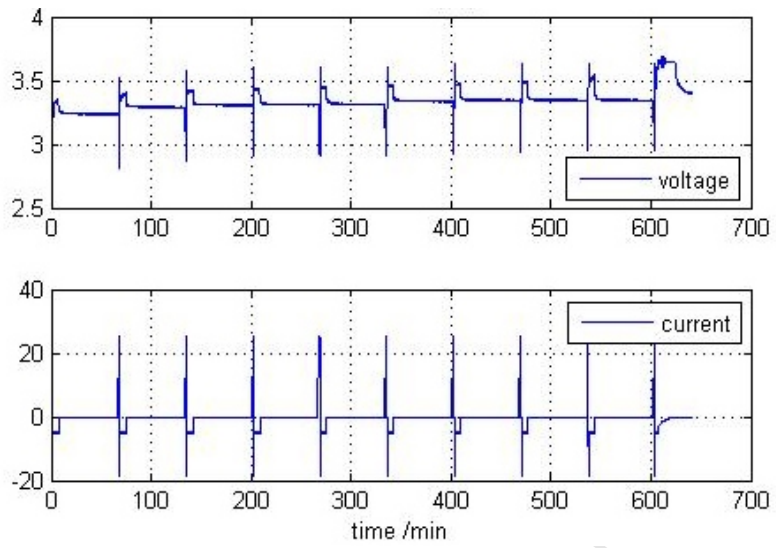

Figure 5: HPPC charging test data
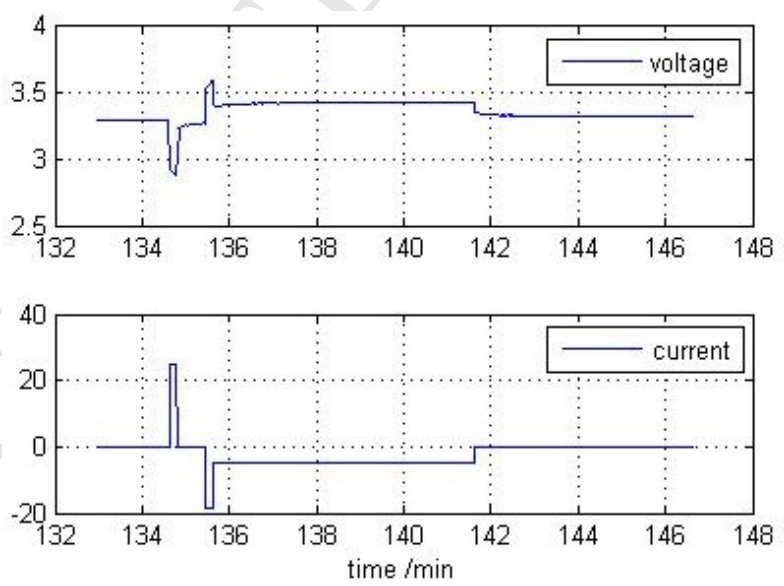

Figure 6: One segment of the HPPC charging test data 

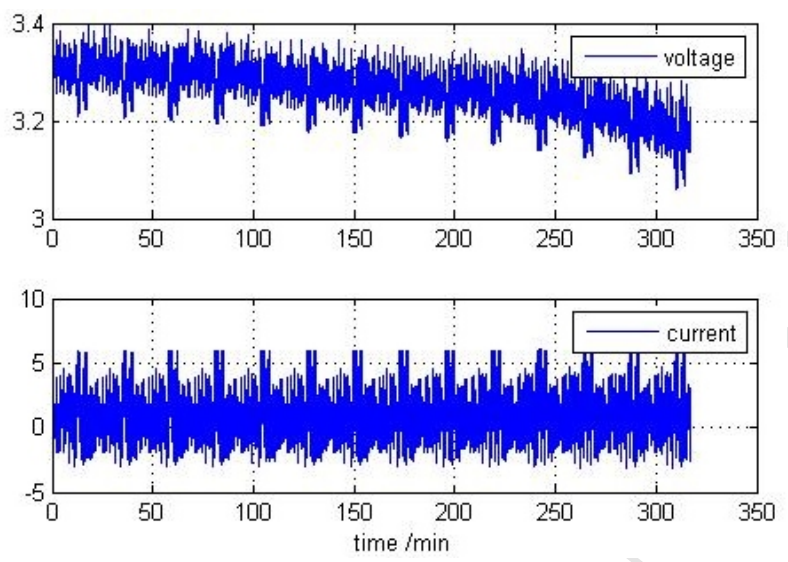

Figure 7: FUDS test data
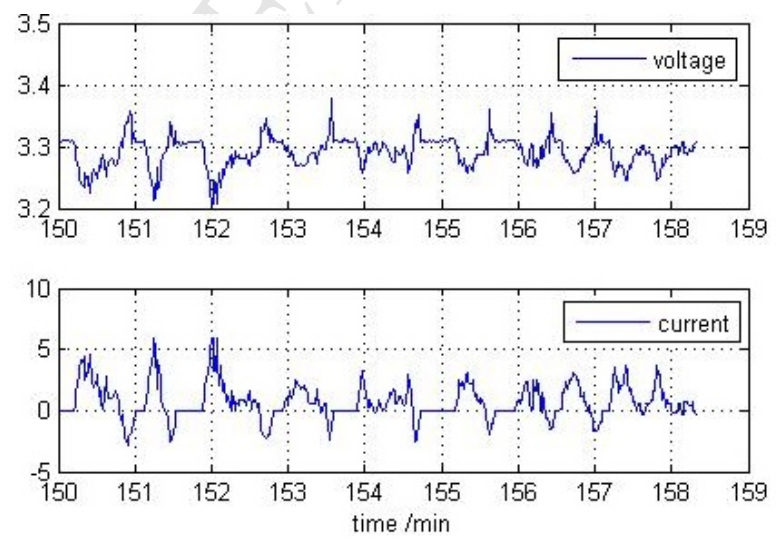

Figure 8: One segment of the FUDS test data 


\section{Modelling}

235 model together with the model parameter identification method are given as follows.

\subsection{Hysteresis model}

As mentioned above, a hysteresis model is essential for the SOC estimation of $\mathrm{LiFePO} 4$ batteries using OCV based method, yet a model that explains the electrochemical causes of the hysteresis effect is too complex to use. Plett [28] proposed a first-order hysteresis model, as follows,

$$
\begin{aligned}
V_{h}(k+1)= & \exp (-|\gamma * i(k)|) * V_{h}(k)+ \\
& (1-\exp (-|\gamma * i(k)|)) * \operatorname{sign}(i(k)) * M_{h}
\end{aligned}
$$

where $V_{h}(k)$ is the hysteresis voltage, $i(k)$ is the current, $\gamma$ a coefficient, and $M_{h}$ is the maximum hysteresis voltage which depends on battery SOC and current rate, and

$$
\operatorname{sign}(i(k))= \begin{cases}1, & \text { if } i(k)>\epsilon \\ -1, & \text { if } i(k)<-\epsilon \\ \operatorname{sign}(i(k-1)), & \text { otherwise }\end{cases}
$$

where $\epsilon$ is a small threshold value.

According to Eq (2), the battery hysteresis voltage is limited between $-M_{h}$ and $M_{h}$, and reaches the limit faster under a larger load current. A constant $M_{h}$ is adopted in this paper.

\subsection{AR model to capture the relaxation effect}

Several equivalent circuit models (ECMs) using a series of RC networks as shown in Fig 2 are widely adopted for capturing the battery relaxation effect, and the model parameters bear certain physical interpretations. For example, the resister $R_{i}$ stands for the battery internal resistance. While ECMs enjoy high interpret-ability, on the other hand, it is this physical interpretation that imposes restrictions on the choice of values for these model parameters. For 
example, all the model parameters for an ECM, e.g., $R_{i}, C_{i}$, have to be positive (i.e., it is unreasonable to assume a negative resistor or negative capacitor in an electric circuit model). This restriction on model parameters will however lead to a limited model fitting performance. Further, some model parameters, such as these $\mathrm{RC}$ network time constants, e.g., $t=R_{i} * C_{i}$, are nonlinear parameters that have to be optimized using complex optimization methods, such as genetic algorithm [29], which will inevitably increase the computational expense. To overcome this limitation and improve the model fitting accuracy, a more general auto-regression $A R(m, n)$ model is adopted in this paper, as shown in Fig9. The governing equation of the relaxation model is

$$
V_{r}(k)=\sum_{i=1}^{m} a_{i} * V_{r}(k-i)+\Sigma_{i=1}^{n} b_{i} * i(k-i)+e(t)
$$

where $V_{r}$ is the battery relaxation voltage, i.e., the over-potential across the $A R$ model, and $e(k)$ is the error term. Although the interpret-ability of the RC networks in the ECM in Fig 2 is lost and the parameters in the AR model, i.e., $a_{i}, b_{i}$ bear no physical meanings, yet on the other hand the generic AR model is capable of capturing unmodelled dynamics of the battery terminal behaviour by the RC networks model.

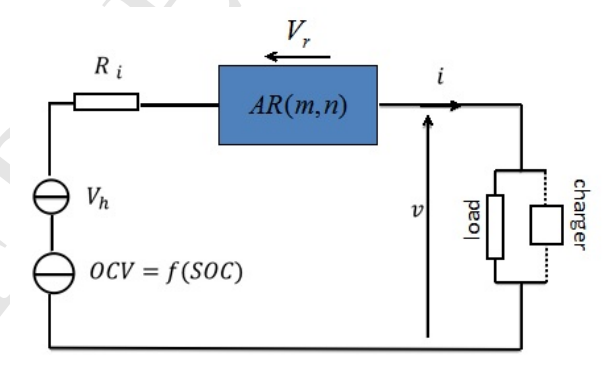

Figure 9: Battery model with $A R(m, n)$ instead of $R C$ networks

\subsection{Model identification}

According to the model in Fig. 9 .

$$
V_{r}(k)=O C V(S O C(k))-V_{h}(k)-v(k)-R_{i} * i(k)
$$


where $v(k)$ is the measured battery terminal voltage, and $R_{i}$ stands for the battery internal resistance.

Let

$$
v_{a}(k)=O C V(S O C(k))-V_{h}(k)-v(k)
$$

and substitute Eq (5) and Eq (4) into Eq (3), we have

$$
\begin{aligned}
v_{a}(k)= & \sum_{i=1}^{m} a_{i} * v_{a}(k-i)+R_{i} * i(k) \\
& +\sum_{i=1}^{n}\left(b_{i}-a_{i} * R_{i}\right) * i(k-i)+e(t)
\end{aligned}
$$

The objective function that is to be optimized is

$$
S S E=\Sigma_{i=1}^{N} e^{2}(t)
$$

while $N$ is the number of data samples used for model training.

Once the measurements of battery terminal voltage and current, i.e., $v(k), i(k)$, become available, $S O C(k)$ can be calculated by Ah method under the laboratory testing conditions, then $O C V(S O C(k))$ by linear interpolation method using the OCV vs SOC curve recorded in Table 1; $V_{h}$ can be calculated by Eq (2) after $M_{h}, \gamma$ are determined. The initial hysteresis voltage, $V_{h}(1)$, depends on the previous load history. In another word, $V_{h}(1)=M_{h}$ if the battery was discharged previously, and $V_{h}(1)=-M_{h}$ if the battery was charged previously.

The model parameters that need to be optimized include

$$
\theta=\left[a, b, \gamma, M_{h}, R_{i}\right]
$$

where

$$
\begin{aligned}
a & =\left[a_{1}, a_{2}, \ldots, a_{m}\right] \\
b & =\left[b_{1}, b_{2}, \ldots, b_{n}\right]
\end{aligned}
$$

This is a non-linear optimization problem, as the $\gamma$ and $M_{h}$ in Eq (2) are non-linear parameters. The Gradient or Hessian information are very difficult to calculate, therefore a heuristic method, namely teaching-learning-based optimization (TLBO) proposed by Rao et al [35], is adopted in this paper for model parameter optimization. TLBO method is a population based method 
that simulates the teaching and learning process in a class. The optimization procedure includes two phases: teacher phase when all the students learn from the teacher (elect the the best student as the teacher), and student phase when the students learn from each other. This optimization algorithm is easy to implement as there is no specific tuning parameters that need to be adjusted by the user.

Note that after $\gamma$ and $M_{h}$ are determined, $v_{a}$ can be calculated as in Eq (5), then according to Eq (6), the rest parameters, i.e., $R_{i}, a, b$, can be optimized using least square method. Therefore, to improve the parameter training efficiency, a hybrid parameter optimization method, namely TLBO plus least square, is used for model training. In another word, the TLBO method is used for non-linear parameter optimization, while the linear parameters are optimzed by the least square method, and the least square method is nested in the TLBO optimization procedure. Therefore, only the two non-linear parameters $\gamma$ and $M_{h}$ need to be optimized by the TLBO method, and the searching space is significantly reduced. Beside, the linear parameters $R_{i}, a, b$ are always kept optimal by using the least square method during the whole model training procedure, which is another advantage of adopting this $A R(m, n)$ model other than the conventional RC circuit model.

The model order selection, i.e, $m, n$, is a trade-off between model complexity and accuracy. The HPPC discharging and charging test data are used for model training, and the FUDS test data are used for model validation. The modelling root mean square error with respect to the model order is shown in Fig 10. As can be seen, as the model order increases from one to three, both the training error and the validation error are reduced noticeably. However, further increasing the model order does not bring about any significant improvement on the model accuracy. Accordingly, $m=n=3$ is selected.

The model parameter optimization procedure is illustrated in Fig 11 . 

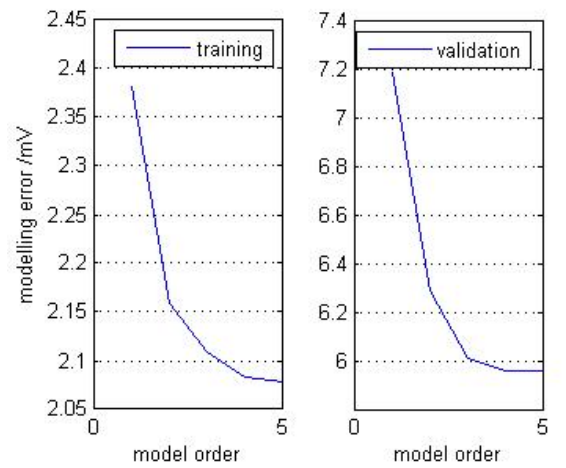

Figure 10: modelling training and validation error with respect to model order

\subsection{Modelling results}

Two test data sets, the HPPC discharging and HPPC charging data, were used for model training, and the FUDS test data was used for model validation. Note that only the test data within $10 \%$ - $70 \%$ SOC range were used for battery model training and validation. The model training results are shown in Fig 12 using HPPC discharging data and Fig 13 using HPPC charging data.

As can be seen, the model outputs match the measured data closely except for a couple of error spikes. The root mean square error is $2.16 \mathrm{mV}$ for HPPC discharging data, and $2.05 \mathrm{mV}$ for HPPC charging data. Consider that the battery voltage changes between $3.0 \mathrm{~V}$ and $3.4 \mathrm{~V}$, the modelling error is less than $0.1 \%$ of the battery voltage.

The FUDS data were used for model validation. The root mean square error is $6.1 \mathrm{mV}$, about $0.2 \%$ of the battery voltage. For illustration purpose, only a part of the validation result is shown in Fig 14. As can be seen, the validation error reminds small except for several spikes.

Those error spikes occur when the load current changes suddenly, or when the load current changes from charging to discharging. These error spikes can be caused by that the simple battery model is not sufficient to capture all the non-linearity of the battery behaviour.

Finally, the model parameters are listed in Table 2. 


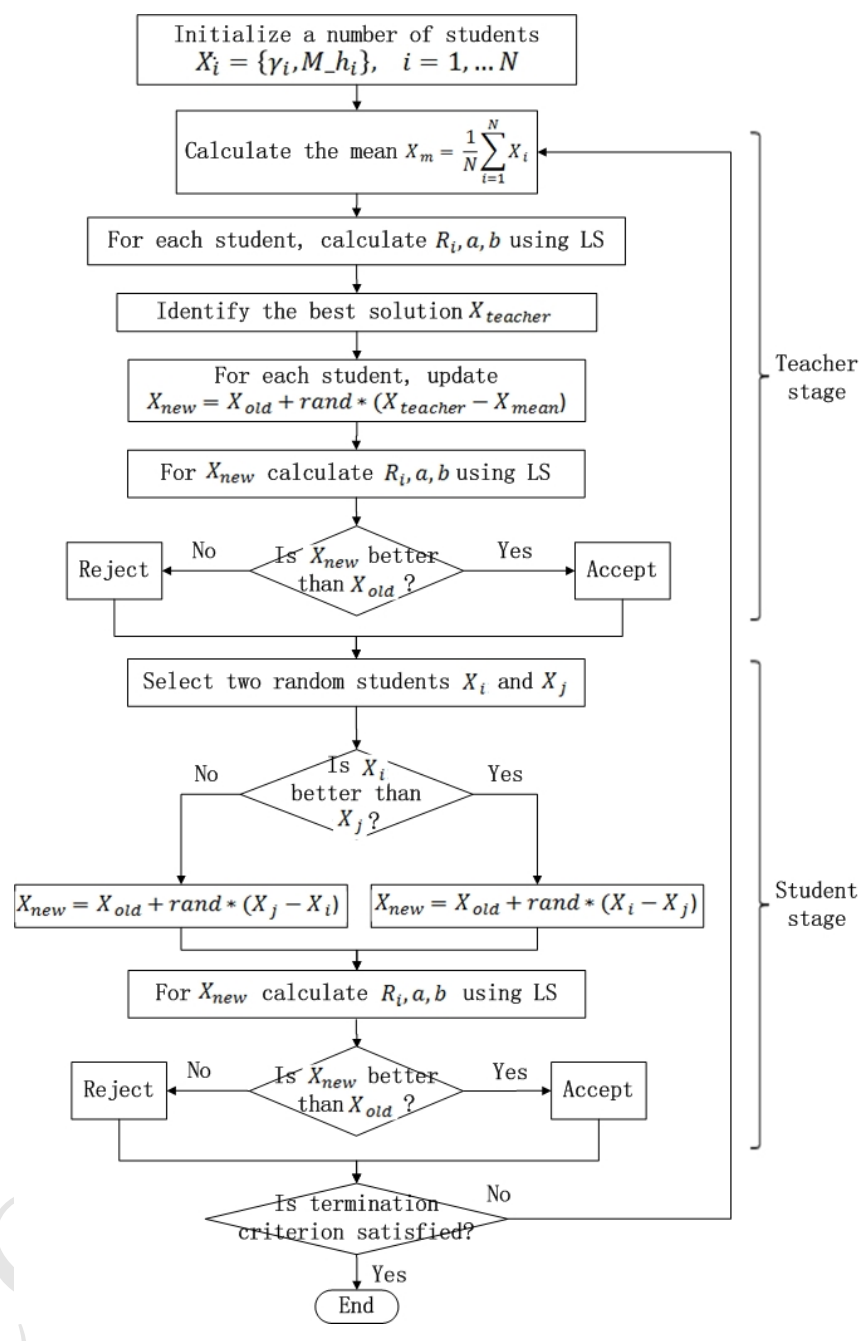

Figure 11: Flowchart of the hybrid parameter optimization method 
Table 2: Identified AR model parameters

\begin{tabular}{cc}
\hline Parameter & Value \\
\hline$\gamma$ & $8.12 \mathrm{E}-4$ \\
$M_{h}$ & 0.0307 \\
$R_{i}$ & 0.0143 \\
$a_{1}$ & 0.4091 \\
$a_{2}$ & 0.3104 \\
$a_{3}$ & 0.2356 \\
$b_{1}$ & $5.965 \mathrm{E}-4$ \\
$b_{2}$ & $2.061 \mathrm{E}-4$ \\
$b_{3}$ & $-2.098 \mathrm{E}-4$ \\
\hline
\end{tabular}
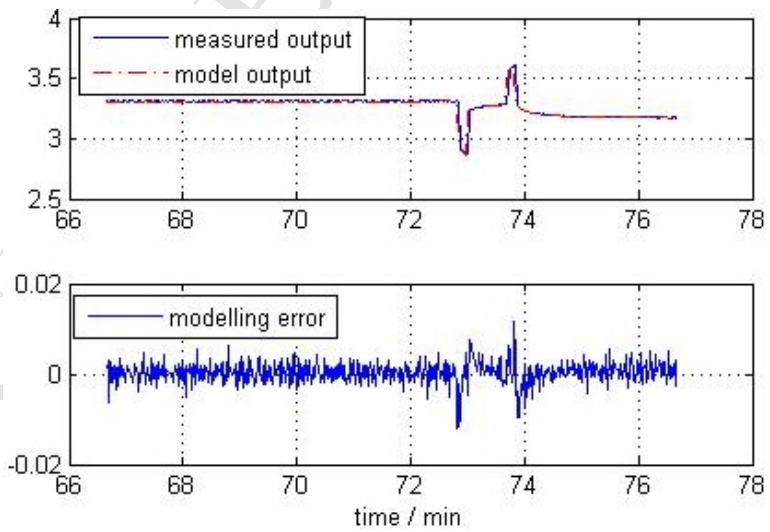

Figure 12: Modelling results using HPPC discharging data 

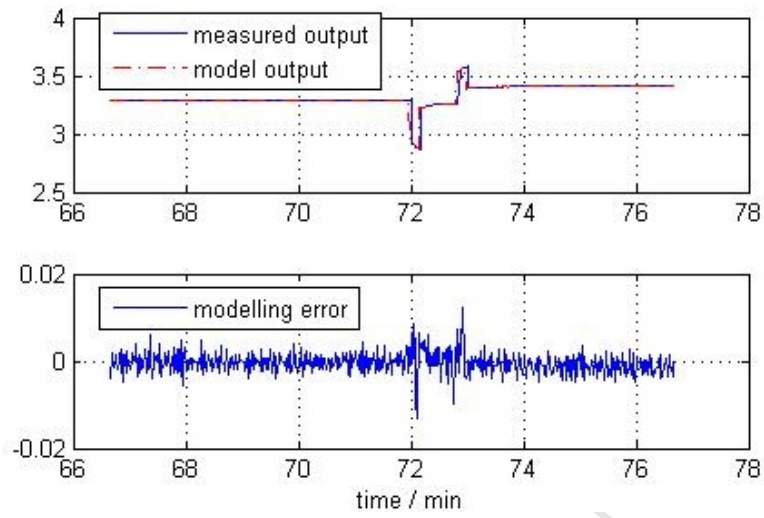

Figure 13: Modelling results using HPPC charging data
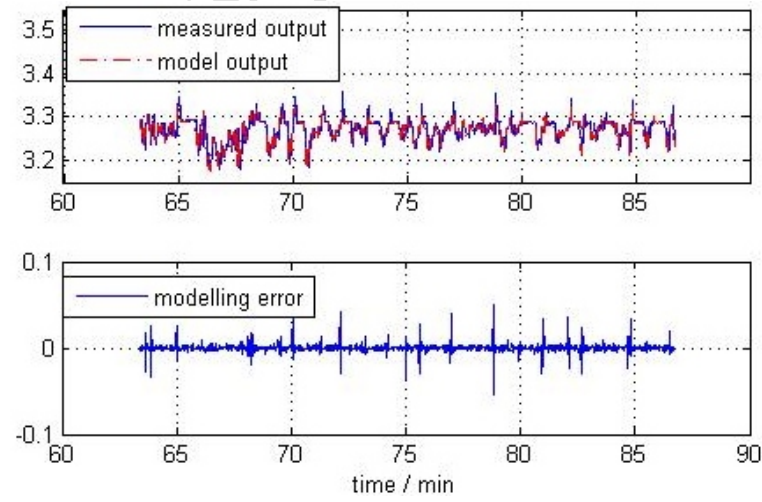

Figure 14: Model validation results using FUDS test data 


\section{Real-time model-based SOC estimation}

Based on the trained model, two types of real-time SOC estimation methods are presented in this section. The first method takes battery OCV as a model parameter which is identified in real-time using WRLS method, then battery SOC is inferred by a lookup table, i.e., Table 1. This method is combined with the Ah method to provide a weighted SOC estimation result. The second one formulates the ECM into a state-space model, and the battery SOC is taken as a state. Then EKF and joint-EKF method are adopted for SOC estimation.

\subsection{Parameter identification based SOC estimation}

The aim of this method is for real-time estimation of the battery OCV. As mentioned before, based on the on-board measured voltage and current signals, Ah method can be used to estimate the SOC; on the other hand, based on the identified model, battery OCV can be inferred in real-time, which will be used to compensate the Ah method.

By substituting Eq (4) into Eq (3) we have

$$
\begin{aligned}
& O C V(S O C(k))-\sum_{i=1}^{m} a_{i} * O C V(S O C(k-i))= \\
& v(k)+V_{h}(k)+R_{i} * i(k)+\sum_{i=1}^{n} b_{i} * i(k-i) \\
& -\sum_{i=1}^{m} a_{i} *\left(v(k-i)+V_{h}(k-i)+R_{i} * i(k-i)\right)+e(k)
\end{aligned}
$$

Now take $O C V(S O C)$ as a time varying parameter, as $\theta_{\text {ocv }}=O C V(S O C(k))$. Apparently, $\theta_{\text {ocv }}$ changes as the discharging continues. If a $5 \mathrm{~A}$ discharging current is applied to a 5 Ah battery, it takes one hour (3600 seconds) for the battery to evolve from fully charged to fully discharged, i.e., for $\theta_{\text {ocv }}$ to change from about $3.4 \mathrm{~V}$ to $3.0 \mathrm{~V}$. Consider that the sampling period used in the test is $T=1 s$, therefore $\theta_{o c v}$ varies slowly with time. Assumed that $\theta_{o c v}=O C V(S O C(k)) \simeq O C V(S O C(k-i)), i=1,2, \ldots, m$, then Eq 9 can be reformulate as

$$
\left(1-\sum_{i=1}^{m} a_{i}\right) * \theta_{\text {ocv }}=u(k)+e(k)
$$


Table 3: Procedure of WRLS

Problem formulation:

$$
y(k)=\varphi(k) * \theta+\eta(k)
$$

where $\varphi(k)$ is the regressing vector, and $\theta$ the parameter vector to be identified, $\eta(k)$ the modelling error

Initialize

$$
\theta(0), P(0)=E\left\{(\theta(0)-\theta)(\theta(0)-\theta)^{T}\right\}
$$

determine the forgetting factor $\lambda$

For $\mathrm{k}=1,2,3 \ldots$,

1) prediction error:

$$
e(k)=y(k)-\varphi^{T}(k) * \theta(k-1)
$$

2) gain:

$$
K=\frac{P(k-1) * \varphi(k)}{\lambda+\varphi(k)^{T} * P(k-1) * \varphi(k)}
$$

3) update

$$
\begin{gathered}
\theta(k)=\theta(k-1)+K * e(k) \\
P(k)=\frac{1}{\lambda}\left(P(k-1)-K * \varphi(k)^{T} * P(k-1)\right)
\end{gathered}
$$

where $u(k)$ stands for all the right hand side terms in Eq (9) excluding $e(k)$. After the battery terminal voltage and current measurement become available, $u(k)$ can be calculated, then $\theta_{o c v}$ can be deduced in real-time.

Since $\theta_{\text {ocv }}$ is a time-varying parameter, the WRLS method is adopted for this parameter estimation. Refer to [36] for details of WRLS method and 37] for a generalize recursive least square parameter identification method. The implementation of WRLS is detailed in Table 3 . In this case, there is only one constant regressor, i.e., $\varphi=\left(1-\sum_{i=1}^{m} a_{i}\right)$ and one parameter, i.e., $\theta_{\text {ocv }}$ to be estimated.

The procedure of SOC estimation using WRLS method is shown in Fig 15 and summarized as follows, 
1. Initialize $S O C_{e}(0)$, the initial estimated SOC; then initialize the WRLS parameters $\theta_{\text {ocv }}$ and $P(0)$ in Table 3 ,

2. For $\mathrm{k}=1,2,3, \ldots$, after new measurements, $v(k), i(k)$ become available:

a) Use Ah method to update battery SOC as follows,

$$
S O C_{i}(k+1)=S O C_{e}(k)-i(k) * T / C_{n}
$$

Note that $S O C_{i}$ is updated based on previously estimated SOC, $S O C_{e}(k)$, $\operatorname{not} S O C_{i}(k)$.

b) Update battery hysteresis voltage $V_{h}(k)$ in Eq (2), then $u(k)$ as in Eq (9). Then apply WRLS method to estimate $\theta_{o c v}(k+1)$ (thus $\left.O C V\left(S O C_{k+1}\right)\right)$ using Eq 10.

c) Based on this estimated $O C V\left(S O C_{k+1}\right)$, a $\mathrm{SOC}$ value can be obtained, i.e., $S O C_{v}(k+1)$, by linear interpolation method using Table 1 .

3) The weighted average between $S O C_{i}(k+1)$ and $S O C_{v}(k+1)$ is used to update battery SOC estimation as follows,

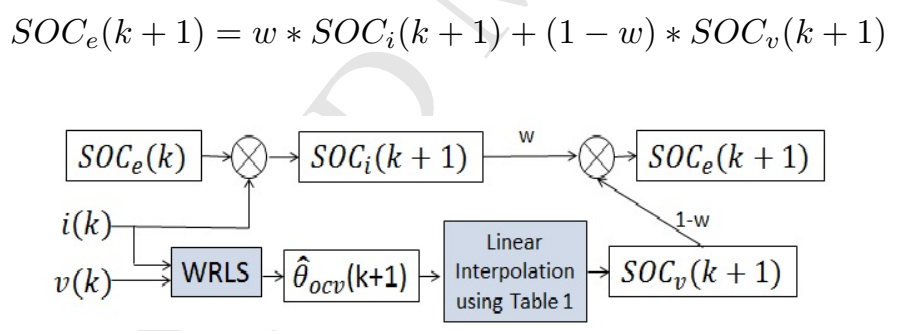

Figure 15: SOC estimation method using WRLS method

\subsection{State-spcae based SOC estimation method}

The transfer function that governs the $A R(m, n)$ model, i.e., Eq (3), can be converted into a state-space formulation as follows,

$$
\begin{aligned}
x_{a r}(k+1) & =A_{a r} * x_{a r}(k)+B_{a r} * i(k) \\
V_{r}(k) & =C_{a r} * x_{a r}(k)
\end{aligned}
$$

where

$$
x_{a r}(k)=\left[x_{1}(k), x_{2}(k), x_{3}(k)\right]^{T}
$$




$$
\begin{gathered}
A_{a r}=\left[\begin{array}{ccc}
0 & 1 & 0 \\
0 & 0 & 1 \\
a_{3} & a_{2} & a_{1}
\end{array}\right] \\
B_{a r}=[0,0,1]^{T} \\
C_{a r}=\left[b_{3}, b_{2}, b_{1}\right]^{T}
\end{gathered}
$$

Combining Eq (2), and Eq (1), and Eq (4), the overall equations governing the model in Fig (9) can be formed into a state-space representation as follows,

$$
\begin{gathered}
x(k+1)=A(k) * x(k)+B(k) \\
v(k)=O C V(S O C(k))-C_{a r} * x_{a r}(k)-V_{h}(k)-R_{i} * i(k)
\end{gathered}
$$

where

$$
\begin{gathered}
x(k)=\left[S O C(k), x_{a r}(k), V_{h}(k)\right]^{T} \\
A(k)=\left[\begin{array}{ccccc}
1 & 0 & 0 & 0 & 0 \\
0 & 0 & 1 & 0 & 0 \\
0 & 0 & 0 & 1 & 0 \\
0 & a_{3} & a_{2} & a_{1} & 0 \\
0 & 0 & 0 & 0 & a_{5,5}
\end{array}\right] \\
B(k)=\left[-T / C_{n} * i(k), 0,0, i(k), b_{5}\right]
\end{gathered}
$$

$a_{5,5}=\exp (-\gamma * a b s(i(k)))$, and $b_{5}=(1-\exp (-\gamma * a b s(i(k)))) * \operatorname{sign}(i(k)) * M_{h}$.

This is a standard state space formulation, with Eq 13 as the state equation, Eq (14) as the output equation, and the battery SOC is one of the model states.

Here,different state estimation methods can be applied for real-time battery SOC estimation, such as extended Kalman filter (EKF) 38], unscented Kalman Filter (UKF) [15, adaptive Kalman filter [39], slide mode observer [40] and $H \infty$ 360 filter 41], etc. 
Kalman filter has been widely applied for on-line state estimation of linear system in various applications. Kalman Filter works in a prediction-correction way. The state is firstly predicted using the state equation. Once a new measurement becomes available, the prediction error is used to correct the state prediction. To extend its application to non-linear systems, extended Kalman filter (EKF) is proposed which firstly linearises the system at the current operating point using first-order Taylor series, then KF can be applied.

As mentioned above, battery internal resistance, $R_{i}$, varies with battery SOC, therefore $R_{i}$ can be taken as a time-varying parameter, or an extra state. Plett [38, 15] proposed using two different methods, joint state estimation or dual state and parameter estimation, to track both battery state and timevarying model parameters in real-time. The joint state estimation method is adopted in this paper, i.e., to treat $R_{i}$ as another state as follows,

$$
R_{i}(k)=R_{i}(k-1)+n_{r}
$$

where $n_{r}$ is assumed to be independent white Gaussian noise, and $E\left\{n_{r} * n_{r}^{T}\right\}=$ $Q_{R}$.

It is straightforward to add $R_{i}$ into the state equation in Eq $\sqrt{13}$ to form an augmented state vector. Then the system parameters will change as follows,

$$
\begin{aligned}
x^{a}(k) & =\left[x^{T}(k), R_{i}(k)\right]^{T} \\
A^{a} & =\operatorname{blkdiag}(A, 1) \\
B^{a} & =[B, 0]^{T}
\end{aligned}
$$

Note that the output equation in Eq (14) keeps unchanged.

In this paper, the $O C V(S O C(k))$ in Eq (14) is a linear-interpolation function using the data recorded in Table1. To apply EKF and joint-EKF for state estimation, the derivative of $O C V(S O C(k))$ with respect to $S O C(k)$ is calculated as follows. The derivative at $15 \%, 25 \%, \ldots$, and $65 \%$ are calculated as the 375 slope of the corresponding segment of the OCV vs SOC curve recorded in Table 1. and the results are listed in Table 4. For example, the derivative at 15\% SOC is calculated as the slope of the OCV vs SOC curve during $10 \%$ to $20 \%$ SOC 


\begin{tabular}{lllllll} 
Table 4: Calculate of $d(O C V(S O C(k)) / d S O C(k)$ \\
\hline SOC $\%$ & 15 & 25 & 35 & 45 & 55 & 65 \\
$d O C V / d S O C$ & 0.484 & 0.293 & 0.0671 & 0.0331 & 0.170 & 0.159 \\
\hline
\end{tabular}

range. The derivative of $O C V(S O C(k))$ with respect to $S O C(k)$ at other SOC points are defined as the linear interpolation of Table 4.

The implementation procedure of EKF is depicted in Table 5.

\section{Results and discussions}

In total, three different SOC estimation methods are compared in this section, one parameter identification based method, i.e., WRLS method, and two state estimation based methods, i.e., EKF and joint-EKF methods. Two different situations are considered, one with correct SOC initial value and the other with $20 \%$ initial error.

\subsection{WRLS based method}

The WRLS-based SOC estimation method is applied to the FUDS test data. Generally speaking, the choice of the forgetting factor in WRLS depends on the change rate of the estimated variables or state. If the variables change slowly with time, a large forgetting factor should be applied as more data samples can be used for generating the estimations. In this study, the average load current of the FUDS test data is about 1 amps, and the sampling time interval is 1 second. So it takes about 900 data samples to reduce the battery SOC by $5 \%$. Thus, the battery OCV, i.e, the estimated parameter changes slowly with time. On the other hand, the weight $w$ in Eq (11) determines the correction rate of the OCV based SOC estimation to the Ah method. Therefore, when the OCV based SOC estimation is reliable, larger weight should be adopted on the OCV estimation. On the other hand, for ranges where the OCV based SOC estimation is not very reliable, e.g., during the flat OCV range, smaller weight should be put on the OCV based SOC correction. Given this above consideration, a variable weight 
Table 5: Procedure of EKF

Problem formulation:

state equation:

$$
x(k+1)=f(x(k), u(k))+w(k)
$$

output equation:

$$
y(k)=g(x(k), u(k))+v(k)
$$

$w(k)$ and $v(k)$ are assumed to be independent Gaussian noise, and

$$
E\left(w(k) w^{T}(k)\right)=Q(k), E\left(v(k) v^{T}(k)\right)=R(k)
$$

Calculate:

$$
\begin{aligned}
A(k) & =\left.\frac{\partial f(x, u)}{\partial x}\right|_{x=\widehat{x}(k)} \\
C(k) & =\left.\frac{\partial g(x, u)}{\partial x}\right|_{x=\widehat{x}_{p}(k)}
\end{aligned}
$$

Initialize

$$
\widehat{x}(0), \Sigma(0)=E\left\{(x(0)-\widehat{x}(0))(x(0)-\widehat{x}(0))^{T}\right\}
$$

For $\mathrm{k}=1,2,3, \ldots$

1) prediction:

$$
\widehat{x}_{p}(k+1)=f(\widehat{x}(k), u(k))
$$

prediction covariance:

$$
\Sigma_{p}(k+1)=A(k) * P(k) * A^{T}(k)+Q(k)
$$

2)correction:

prediction error:

$$
e(k+1)=y(k+1)-g\left(\widehat{x}_{p}(k+1), u(k+1)\right)
$$

gain:

$$
\begin{gathered}
K=\Sigma_{p}(k+1) * C^{T}(k+1) * \\
\left(C(k+1) * \Sigma_{p}(k+1) * C^{T}(k+1)+R(k)\right)^{-1}
\end{gathered}
$$

update:

$$
\begin{gathered}
\widehat{x}(k+1)=\widehat{x}_{p}(k+1)+K * e(k+1) \\
\Sigma(k+1)=(I-K * C(k+1)) * \Sigma_{p}(k+1)
\end{gathered}
$$


is preferable for the method combining both Ah method with OCV based SOC estimation. In the following section, we first choose $\mathrm{w}=0.95$, and then discuss the impact of variable weight $w$ on the estimation performance.

The estimation results with a $20 \%$ initial SOC estimation error are depicted in Fig 16 and with correct initial SOC estimation in Fig 17.
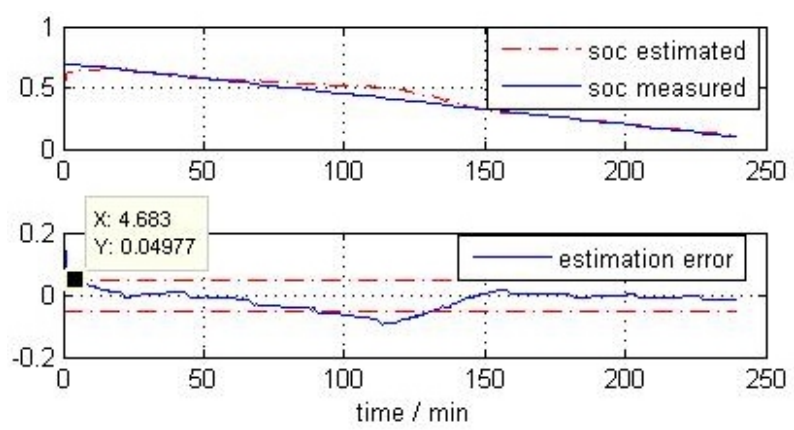

Figure 16: SOC estimation results using WRLS with $20 \%$ initial estimation error
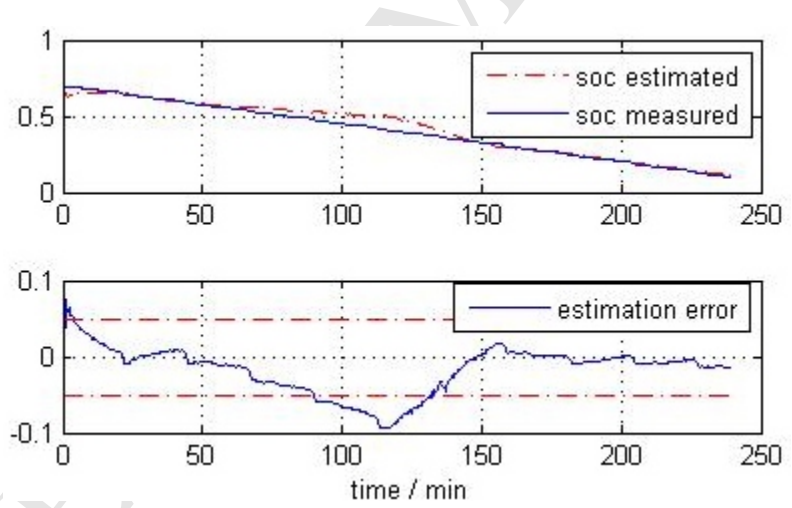

Figure 17: SOC estimation results using WRLS with correct initial SOC

As can be seen in Fig 16, the SOC estimation error converges from $20 \%$ to within $5 \%$ in about five minutes, which proved the effectiveness of this OCVbased compensation method. The error at the starting stage in Fig 17 might be caused by the incorrect initial guess of the hysteresis voltage, i.e., $V_{h}(0)$, which faded away in couple of minutes.

However, in both Fig 16 and Fig 17, large SOC estimation errors occur 
during $50 \%-30 \%$ SOC range. It is because the battery OCV vs SOC curve is so flat during this SOC range that it is very difficult to provide correct SOC estimation using the noisy estimated battery OCV value. As can be seen in Table 1, battery OCV only drops $3.4 \mathrm{mV}$ and $6.7 \mathrm{mV}$ when battery SOC is reduced from $50 \%$ to $40 \%$, and from $40 \%$ to $30 \%$, respectively.

Under the laboratory test condition, the battery SOC can be directly calculated by the Ah method, then the battery OCV can be obtained by linear interpolation method using the OCV vs SOC relation as listed in Table 1, and the resultant $\mathrm{OCV}$ data is called 'real $O C V$ ' in this paper. Note that the 'real $O C V^{\prime}$ is calculated using the average of the charging and discharging battery OCV values. The difference between the estimated battery OCV using WRLS method and the 'real $O C V$ ' is depicted in Fig. 18. The battery SOC is correctly initialized.

As it is shown in Fig 18, the estimated battery OCV is very close to the 'real $O C V$ '. As a matter of fact, the estimated OCV error keeps below $5 \mathrm{mV}$ for most of the time. However, because of the flat slope of the OCV vs SOC curve, $5 \mathrm{mV}$ error in the estimated OCV can cause up to $7.5 \%$ SOC estimation error during the $30 \%-40 \%$ SOC range, and more than $10 \%$ SOC estimation error during $40 \%$ - 50\% SOC range using linear interpolation of Table 1 . On the other hand, $5 \mathrm{mV}$ OCV estimation error can only cause about $1 \%$ SOC estimation error during $10 \%-20 \%$ SOC range.

A practical way to tackle this problem is to reduce the weight of $S O C_{v}$ in Eq 435 (11) when $S O C_{v}$ lies between $30 \%$ and $50 \%$ SOC, i.e., reduce the compensation effect when the OCV-based correction is not so reliable. Let $w=1$ in Eq (11) when $30 \%<S O C_{v}<50 \%$, which means that when the estimated OCV falls between $30 \%$ and $50 \%$ SOC range (i.e., $S O C_{v}$ is not reliable), the OCV-based correction is avoided. Note that a constant estimation error is expected when ${ }_{440} w=1$. The new SOC estimation results are depicted in Fig 19 with $20 \%$ initial SOC estimation error and in Fig 20 where the estimated SOC is correctly initialized. As can be seen, the large SOC estimation errors during $30 \%$ to $50 \%$ SOC range in Fig 16 and Fig 17 are successfully removed and replaced with 
constant estimation error as shown in Fig 19 and Fig 20.

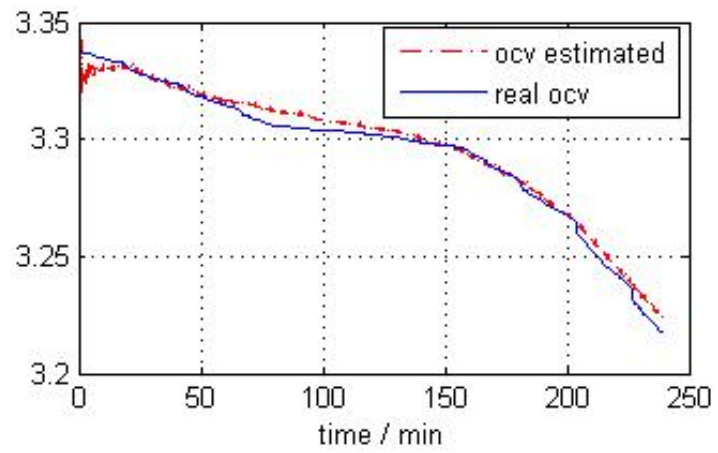

Figure 18: Estimated OCV using WRLS and the 'real OCV' with correct initial SOC
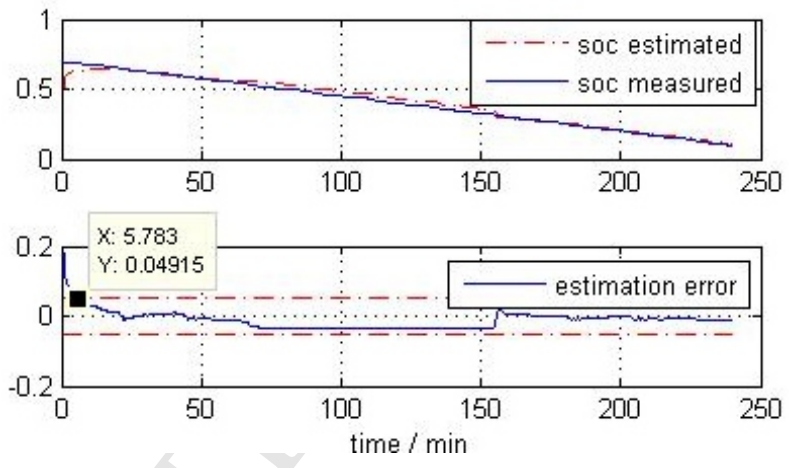

Figure 19: SOC estimation results using WRLS after changing the weight during $30 \%$ to $50 \%$ SOC range with $20 \%$ initial SOC estimation error

The SOC estimation results using the EKF method are shown in Fig 21 and Fig 22. In Fig 21 the initial SOC estimation error is 20\%. As can be seen, the estimated SOC converges to within $5 \%$ SOC error in about 5 minutes, and the error remains within $5 \% \mathrm{SOC}$ afterwards. The large estimation errors caused by the flat OCV vs SOC curve during $50 \%$ to $30 \%$ SOC range, as shown in Fig 16 and Fig 17, are greatly suppressed. The reason is that the estimated SOC has already converged to the corrected SOC before reaching 50\% SOC level. The 

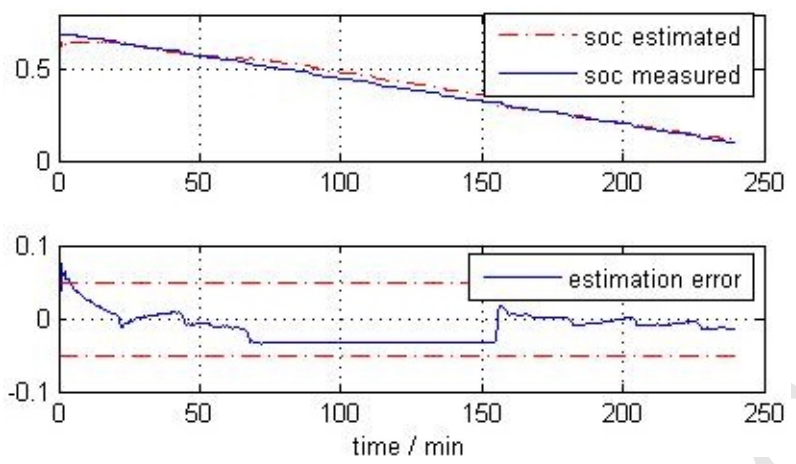

Figure 20: SOC estimation results using WRLS after changing the weight during $30 \%$ to $50 \%$ SOC range with correct initial SOC estimation

change of the gain $K$ in the EKF algorithm shown in Table 5 i.e., $\|K\|_{2}$ which determines the correction weight, is depicted in Fig 23. As can be seen, after the estimation error converges, the amplitude of $K$ falls quickly to a very low value.

In Fig 22, the estimated SOC is correctly initialized. It shows that the SOC estimation error remains quite small, except for one segment at the end of the discharging period. This problem will be tackled using the joint-EKF method.
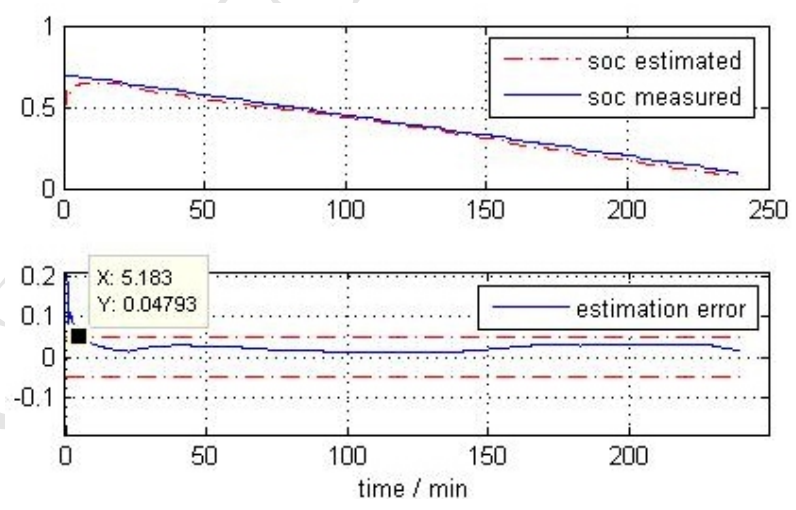

Figure 21: SOC estimation results using EKF with 20\% initial estimation error 

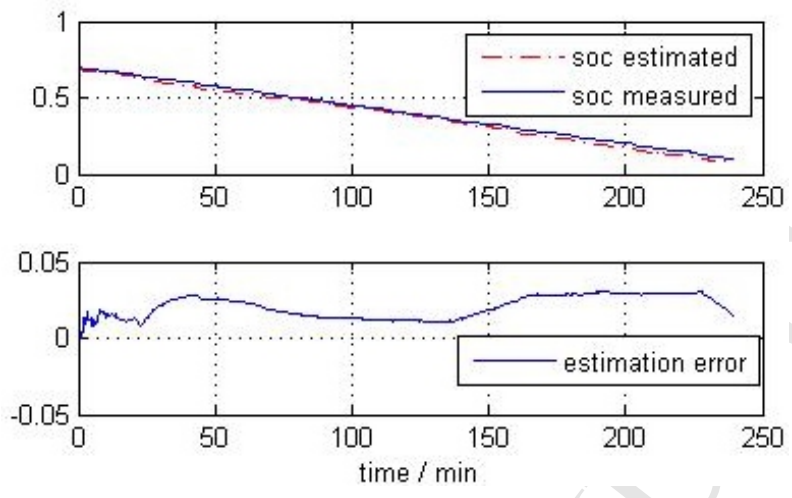

Figure 22: SOC estimation results using EKF with correct initial SOC

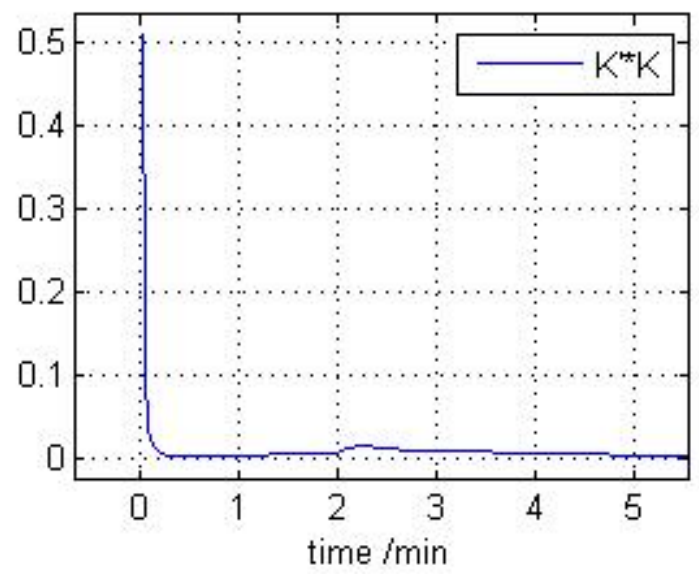

Figure 23: Amplitude of the gain $K$ 
As discussed in the previous section, the battery internal resistance, $R_{i}$ can be taken as another state, thus forming the augmented state vector as shown in 15. Then the EKF method can be applied for SOC estimation. The results are depicted in Fig 24 with $20 \%$ initial SOC estimation error and in Fig 25 where the estimated SOC is correctly initialized. It shows that with a $20 \%$ initial SOC estimation error, it takes less than one minute for the estimated error to fall below $5 \%$ and remain within $5 \%$ afterwards. In both cases, the estimation errors are very small.

The large SOC estimation error at the end of discharge in Fig 22 is successfully suppressed in Fig 25 by introducing $R_{i}$ as another state. Besides, the convergence speed using joint-EKF method (about one minute) is much faster than EKF method (about 5 minutes). The identified battery internal resistance, $R_{i}$, as an extra state is shown in Fig 26. It shown that $R_{i}$ increased notably at the end of discharging when the battery SOC is reduced to less than $30 \%$, which coincides with laboratory observations that the battery internal resistance will increase as it approaches the end of discharging.
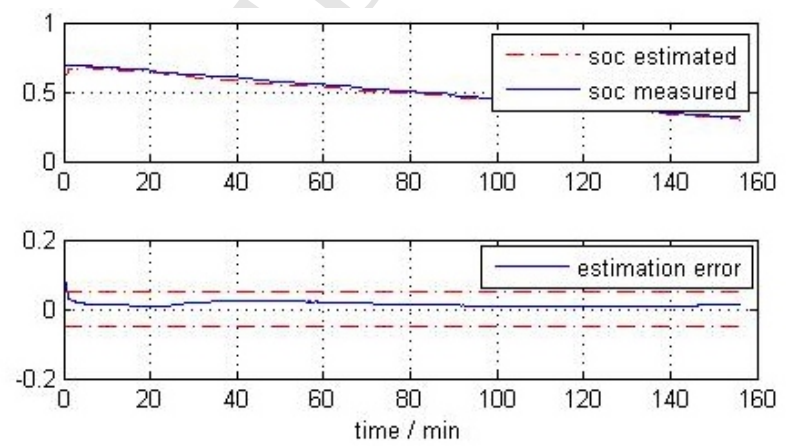

Figure 24: SOC estimation results using joint-EKF with incorrect initial SOC

\subsection{Summary of the results}

Finally, the above SOC estimation results using different methods are summarized in Table 6. As can be seen, the joint-EKF method prevails at both 

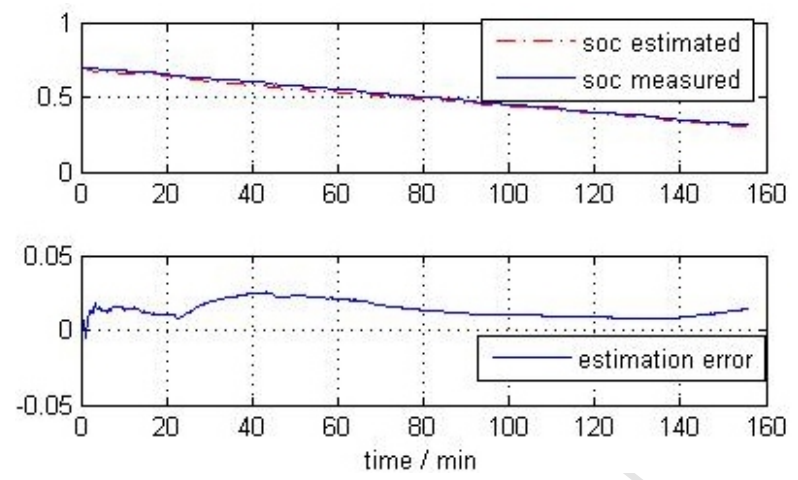

Figure 25: SOC estimation results using joint-EKF with correct initial SOC

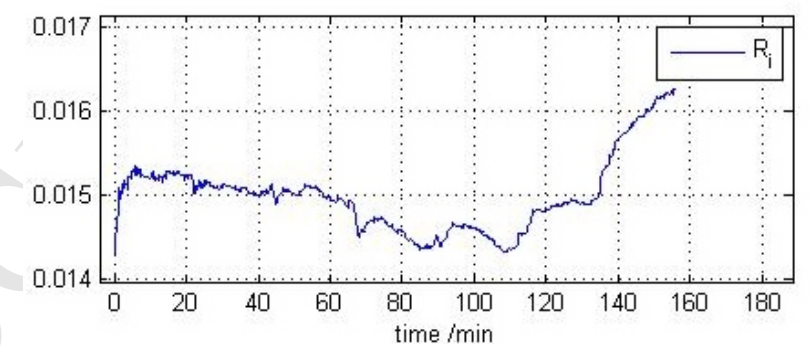

Figure 26: The identified battery internal resistance 
Table 6: Root mean square of SOC estimation error of different methods

\begin{tabular}{l|llll}
\hline & WRLS & $\begin{array}{l}\text { WRLS } \\
\text { changing } \\
\text { weight }\end{array}$ & EKF & joint \\
& \multicolumn{4}{|c}{ EKF } \\
\hline $20 \%$ ini- & $3.67 \%$ & $2.78 \%$ & $2.81 \%$ & $\mathbf{1 . 6 3 \%}$ \\
tial error & & & & \\
\hline no initial & $3.51 \%$ & $2.36 \%$ & $2.17 \%$ & $\mathbf{1 . 4 8 \%}$ \\
error & & & & \\
\hline
\end{tabular}

cases, i.e., with and without initial SOC estimation error. The performance of the WRLS method with changing weight is close to that of the EKF method. The WRLS method alone achieved the worse performance in both cases. Since the OCV-based method is used to compensate for the Ah method, it is natural to avoid using it for certain SOC ranges where the OCV-based SOC estimation is not so reliable, i.e., where the slope of the OCV vs SOC curve is very flat.

The SOC estimation accuracy of the proposed methods are comparable to or better than the published results on various Lithium batteries (e.g., LiB, $\mathrm{LiFePo} 4$ batteries), for most of which the SOC estimation errors are around $2 \%$ [15, 38, 12, 14, 34, 39. Higher SOC estimation accuracy can also be achieved 40 by using a more detailed battery model, such as by taking into consideration of the temperature effect and the rate-dependent columbic efficiency [11, which are however not considered in this paper.

It should be noted that although the results presented in this study are obtained from simulations using experimentally measured data, however, once the battery model is trained off-line using experimental data, the computational complexity of the SOC estimation methods proposed in this paper is quite low, and in this paper both the extended Kalman Filter method and the WRLS method are presented in a recursive formula, which are designed for on-line applications. Further, the methods presented in this paper depend only on the on-board measurable signals, such as voltage and current signals. Therefore, 
the methods proposed in this paper are for real-time on-board applications.

\section{Conclusions}

Real-time accurate SOC estimation is of great importance for the battery management system in EV/HEV applications. Different SOC estimation meth-

ods are firstly presented in this paper, including direct measurement methods and model-based estimation methods. An auto-regression battery model is then proposed to reproduce the battery terminal behaviour together with a non-linear complementary model to capture the battery hysteresis effect. The model parameters are optimized using a hybrid optimization method combining TLBO and least square method. Based on the off-line trained model, two different real-time model-based SOC estimation methods for Lithium-ion batteries are presented, one based on model parameter identification using WRLS method and another based on state estimation using EKF method. Considering that the battery internal resistance changes with battery SOC, joint-EKF method is adopted for both parameter and state estimation to improve the SOC estimation performance. The proposed methods are compared using test data collected on a LiFePo4 battery cell. Two different cases are considered, with and without initial SOC estimation error. The estimation results confirmed the effectiveness of the modelling method and the model-based SOC estimation methods, in particular the joint-EKF method.

Note that the modelling and SOC estimation methods proposed in this paper are data-driven methods using on-board measured signals such as terminal voltage and current, and involve no specific battery chemistries, therefore they are generic for wide applications. In particular, this paper addresses the two most challenging issues in estimating the SOC of these battery types, i.e. flat OCVversus-SOC curve for some SOC ranges, and the hysteresis nonlinearity during the charging and discharging phases. Therefore, although only the LiFePo4 battery is tested in this paper, the methods can be applied to other types of lithium batteries as well as other cathode-based batteries, such as NMP and LiCoO2 
[5] S. Piller, M. Perrin, A. Jossen, Methods for state-of-charge determination and their applications, Journal of power sources 96 (1) (2001) 113-120. 
[6] W. Chang, The state of charge estimating methods for battery: a review, ISRN Applied Mathematics 2013.

[7] N. Watrin, B. Blunier, A. Miraoui, Review of adaptive systems for lithium batteries state-of-charge and state-of-health estimation, in: Transportation Electrification Conference and Expo (ITEC), IEEE, 2012, pp. 1-6.

[8] V. Prajapati, H. Hess, E. William, V. Gupta, M. Huff, M. Manic, F. Rufus, A. Thakker, J. Govar, A literature review of state-of-charge estimation techniques applicable to lithium poly-carbon monoflouride (li/cfx) battery, in: Power Electronics (IICPE), 2010 India International Conference on, IEEE, 2011, pp. 1-8.

[9] J. Li, J. Klee Barillas, C. Guenther, M. A. Danzer, A comparative study of state of charge estimation algorithms for lifepo 4 batteries used in electric vehicles, Journal of Power Sources 230 (2013) 244-250.

[10] H. He, H. Qin, X. Sun, Y. Shui, Comparison study on the battery soc estimation with ekf and ukf algorithms, Energies 6 (10) (2013) 5088-5100.

[11] M.-H. Chang, H.-P. Huang, S.-W. Chang, A new state of charge estimation method for lifepo4 battery packs used in robots, Energies 6 (4) (2013) 20072030.

[12] S. Lee, J. Kim, J. Lee, B. Cho, State-of-charge and capacity estimation of lithium-ion battery using a new open-circuit voltage versus state-of-charge, Journal of Power Sources 185 (2) (2008) 1367-1373.

[13] Lifepo4 battery temperatures test (Jun. 2014). URL http://www.bestgopower.com/technology/documents/ temperature-test.html

[14] F. Baronti, G. Fantechi, L. Fanucci, E. Leonardi, R. Roncella, R. Saletti, S. Saponara, State-of-charge estimation enhancing of lithium batteries through a temperature-dependent cell model, in: Applied Electronics (AE), 2011 International Conference on, IEEE, 2011, pp. 1-5. 
[15] G. L. Plett, Sigma-point kalman filtering for battery management systems of lipb-based hev battery packs: Part 2: Simultaneous state and parameter estimation, Journal of Power Sources 161 (2) (2006) 1369-1384.

[16] M. A. Roscher, O. Bohlen, J. Vetter, Ocv hysteresis in li-ion batteries including two-phase transition materials, International Journal of Electrochemistry 2011.

[17] Y. Zhu, C. Wang, Strain accommodation and potential hysteresis of lifepo4 cathodes during lithium ion insertion/extraction, Journal of Power Sources 196 (3) (2011) 1442-1448.

[18] T. Sasaki, Y. Ukyo, P. Novák, Memory effect in a lithium-ion battery, Nature materials 12 (6) (2013) 569-575.

[19] L. Pei, R. Lu, C. Zhu, Relaxation model of the open-circuit voltage for state-of-charge estimation in lithium-ion batteries, IET Electrical Systems in Transportation 3 (4) (2013) 112-117.

[20] S. Rodrigues, N. Munichandraiah, A. Shukla, A review of state-of-charge indication of batteries by means of ac impedance measurements, Journal of Power Sources 87 (1) (2000) 12-20.

[21] F. Orsini, M. Dollé, J.-M. Tarascon, Impedance study of the li/electrolyte interface upon cycling, Solid State Ionics 135 (1) (2000) 213-221.

[22] How to measure state-of-charge (Feb. 2014). URL http://batteryuniversity.com/learn/article/ howtomeasurestateof charge

[23] S. Buller, M. Thele, E. Karden, R. W. De Doncker, Impedance-based nonlinear dynamic battery modeling for automotive applications, Journal of Power Sources 113 (2) (2003) 422-430.

[24] D. Di Domenico, G. Fiengo, A. Stefanopoulou, Lithium-ion battery state of charge estimation with a kalman filter based on a electrochemical model, 
in: Control Applications, IEEE International Conference on, IEEE, 2008, pp. $702-707$.

[31] C. Bo, B. Zhifeng, C. Binggang, State of charge estimation based on evolutionary neural network, Energy conversion and management 49 (10) (2008) 2788-2794. 
[32] A. J. Salkind, C. Fennie, P. Singh, T. Atwater, D. E. Reisner, Determination of state-of-charge and state-of-health of batteries by fuzzy logic methodology, Journal of Power Sources 80 (1) (1999) 293-300.

[33] T. Hansen, C.-J. Wang, Support vector based battery state of charge estimator, Journal of Power Sources 141 (2) (2005) 351-358.

[34] W. Junping, G. Jingang, D. Lei, An adaptive kalman filtering based state of charge combined estimator for electric vehicle battery pack, Energy Conversion and Management 50 (12) (2009) 3182-3186.

[35] R. Rao, V. Savsani, D. Vakharia, Teaching-learning-based optimization: A novel method for constrained mechanical design optimization problems, Computer-Aided Design 43 (3) (2011) 303-315.

[36] L. Ljung, System identification: theory for the user, Cliffs, N.J.: Prentice Hall, 1987.

[37] M. Verbrugge, B. Koch, Generalized recursive algorithm for adaptive multiparameter regression application to lead acid, nickel metal hydride, and lithium-ion batteries, Journal of The Electrochemical Society 153 (1) (2006) A187-A201.

[38] G. L. Plett, Extended kalman filtering for battery management systems of lipb-based hev battery packs: Part 3. state and parameter estimation, Journal of Power sources 134 (2) (2004) 277-292.

[39] F. Sun, X. Hu, Y. Zou, S. Li, Adaptive unscented kalman filtering for state of charge estimation of a lithium-ion battery for electric vehicles, Energy 36 (5) (2011) 3531-3540.

[40] I.-S. Kim, A technique for estimating the state of health of lithium batteries through a dual-sliding-mode observer, Power Electronics, IEEE Transactions on 25 (4) (2010) 1013-1022. 
[41] F. Zhang, G. Liu, L. Fang, H. Wang, Estimation of battery state of charge with observer: Applied to a robot for inspecting power transmission lines, Industrial Electronics, IEEE Transactions on 59 (2) (2012) 1086-1095. 


\section{Highlights}

- An auto-regression battery model is built considering hysteresis nonlinearity

- A hybrid model training method combining TLBO and least square is proposed

- WRLS and joint-EKF approaches are used for real-time model-based SOC estimation

- Flat OCV problem is tackled by combining WRLS method with coulomb counting 